\title{
Indirect costs of inflammatory bowel diseases: Crohn's disease and ulcerative colitis. A systematic review
}

Paweł Kawalec

Drug Management Department, Institute of Public Health, Faculty of Health Sciences, Jagiellonian University Medical College, Krakow, Poland

Submitted: 6 August 2014

Accepted: 10 November 2014

Arch Med Sci 2016; 12, 2: 295-302

DOI: 10.5114 /aoms.2016.59254

Copyright $\odot 2016$ Termedia \& Banach

\section{Abstract}

Introduction: Crohn's disease and ulcerative colitis are lifelong illnesses which have a significant impact on quality of life and personal burden through a reduction in the ability to work, sick leave and restrictions of leisure time. The aim of this study was to conduct a systematic review of the indirect costs of Crohn's disease and ulcerative colitis.

Material and methods: The search was carried out in Medline, EMBASE, the Centre for Reviews and Dissemination, and reference lists of identified articles and reference lists of identified articles were also handsearched. All costs were adjusted to 2013 USD values by using the consumer price index and purchasing power parity. Identified studies were then analysed in order to assess their heterogeneity and possibility of inclusion in the meta-analysis. Results: Eleven of the identified publications presented indirect costs of Crohn's disease or ulcerative colitis. The range of estimated yearly indirect costs per patient was large, from \$1 159.09 for loss of earnings to $\$ 14135.64$ for lost productivity and sick leave for Crohn's disease. The values for ulcerative colitis ranged from $\$ 926.49$ to $\$ 6583.17$. Because of the imprecise definition of methods of indirect cost calculations as well as heterogeneity of indirect cost components, a meta-analysis was not performed. Conclusions: The indirect costs of ulcerative colitis seem to be slightly lower than in the case of Crohn's disease. A small number of studies referring to indirect costs of Crohn's disease and ulcerative colitis were identified, which indicates the need to conduct further investigations on this problem.

Key words: Crohn's disease, ulcerative colitis, indirect costs, productivity loss.

\section{Introduction}

Crohn's disease and ulcerative colitis are two idiopathic inflammatory bowel disorders. The clinical course of Crohn's disease and ulcerative colitis is unpredictable, marked by alternating periods of exacerbation and remission. Ulcerative colitis is restricted to the colon [1], but Crohn's disease can affect the entire gastrointestinal tract, from the mouth to the anus, and patients frequently present with abdominal pain, fever, and clinical signs of bowel obstruction or diarrhoea with the passage of blood or mucus, or both [1, 2].

Therapy of Crohn's disease and ulcerative colitis is directed at quickly resolving symptoms and subsequently maintaining symptom-free periods. Lifelong medical treatment is required, and often innovative biologic therapy is necessary, which is effective in the majority of patients $[3,4]$ but expensive and difficult to achieve in many countries.

\author{
Corresponding author: \\ Paweł Kawalec MD, PhD \\ Drug Management \\ Department \\ Institute of Public Health \\ Faculty of Health Sciences \\ Jagiellonian University \\ Medical College \\ 20 Grzegorzecka St \\ 31-531 Krakow, Poland \\ Phone: +48 124332801 \\ E-mail: pawel.kawalec@ \\ uj.edu.pl
}


For many patients, Crohn's disease imposes a substantial personal burden, with unpredictable fluctuating symptoms, time off work, the need for expensive drugs, or surgery and multidisciplinary care. With a prevalence of about $0.1 \%(0.007 \%$ to $0.27 \%$ for Crohn's disease and $0.008 \%$ to $0.35 \%$ for ulcerative colitis) [5] in many developed countries Crohn's disease also places a major burden on public health-care resources.

Crohn's disease and ulcerative colitis are lifelong diseases, and have a significant impact on quality of life and personal burden through reduction in the ability to work, long-term and shortterm interruptions in work, restrictions of leisure time, and out-of-pocket expenses (e.g. travel, carers). For all the above reasons the indirect costs of Crohn's disease and ulcerative colitis are expected to have a significant role in the disease burden.

The aim of this review is to assess the indirect costs of Crohn's disease and ulcerative colitis and to define their main components. The conclusions resulting from this study can constitute the basis for further research.

\section{Material and methods}

\section{Indirect costs}

Indirect costs or productivity losses are the labour earnings that are forgone as a result of an adverse health outcome. The decreased productivity can be a result of illness, death, side effects, or time spent receiving treatment. Indirect costs include the lost earnings and productivity of both patients and the family members who take care of them. They consist of three major components: absence from paid work, including sick leave, early retirement and reduced employment (or unemployment) (absenteeism), reduced productivity of paid work (presenteeism), and reduced opportunities for unpaid activities (loss of leisure) [6].

Absenteeism refers to the number of days on sick leave, and early leaving of the labour market due to sickness (e.g. early retirement or early death). Presenteeism refers to a situation when a sick person is present at work but their productivity is lower than average due to disease.

Indirect costs may be difficult to quantify because of a lack of quality data, but they often represent a significant percentage of the total cost associated with many diseases [7].

Each category of indirect costs can be calculated using one of two methods: the human capital approach and the friction cost approach. The human capital approach (HCA) converts the gross income which will not be obtained in the future due to disease into the real costs from a social perspective. The HCA is based on the assumption that work not done due to disease is a decrease of human capital and is a burden to society. The friction cost approach (FCA) takes into account productivity losses until a new person is employed as a substitute for the sick one. The FCA is based on the assumption that society can replace a sick person in order to prevent productivity losses [7].

\section{Literature search}

A systematic literature review was conducted to obtain studies concerning the indirect costs of Crohn's disease and ulcerative colitis. Articles written in English, published from 1990 to March 2014 (cut-off date: $6^{\text {th }}$ March 2014), were included. To include all relevant studies, a search of Medline (using MeSH Terms), EMBASE (using Emtree), and the Centre for Reviews and Dissemination (CRD) databases was conducted. Search terms included: (inflammatory bowel disease OR Crohn's OR Crohn OR (ulcerative AND (disease OR colitis))) AND ((indirect OR productivity OR economic) AND (cost OR costs OR loss OR burden OR (human AND capital))). No date/time/country filters were used. We considered original studies and systematic reviews as well as economic evaluations written in English. Records were excluded if they did not present any kind of indirect costs, or referred to illness other than Crohn's disease or ulcerative colitis. Firstly, papers were excluded if they were not concerned with Crohn's disease or ulcerative colitis, based on titles. Subsequent papers were excluded if they were irrelevant to the indirect costs, based on abstracts. The remaining papers were assessed based on full texts. Additionally, reference lists of identified studies were reviewed to include all relevant studies. Original sources of information concerning the indirect costs of Crohn's disease and ulcerative colitis were included. Economic analysis of particular technologies, such as cost-utility studies, were excluded as they usually concern hypothetical groups of patients (e.g. for Markov modelling) and mostly do not focus on overall cost estimates. Additionally, abstracts and posters were excluded as they do not provide sufficient data and should not be included in a systematic literature search. This systematic review was conducted in accordance with the PRISMA Statement to ensure a proper record selection process.

\section{Synthesis of literature}

All identified articles concern the indirect costs of inflammatory bowel disease, especially Crohn's disease and ulcerative colitis. Data from eligible articles were collected, including the author's name, publication year, reference year for cost, region, number of patients, study method, components of indirect costs, and estimated indirect cost 
per patient. In order to achieve comparability of results across the studies, the author attempted to break down the indirect expenses identified in particular studies into the same categories.

In order to facilitate the comparison of data between the studies, the costs were converted into annual rates. If costs per patient were not provided, the appropriate values were estimated using data reported in the original paper. The consumer price index (CPI) was used to adjust costs to 2013 values, and then all costs were converted to USD purchasing power parity (PPP). If the cost reference year included a range of dates, the lesser value was chosen.

Studies were assessed in terms of the compatibility of the population, endpoints, methodology and presented results. All calculations were done using Microsoft Excel 2007 and OpenMetaAnalyst (for the purpose of meta-analysis).

Estimates of the indirect costs of inflammatory bowel disease were also presented, as the majority of the participants in these studies have Crohn's disease or ulcerative colitis.

\section{Results}

In summary, 166 titles concerning the indirect costs of Crohn's disease or ulcerative colitis were identified in Medline, 161 in EMBASE, and 40 in CRD. After eliminating repetitive records, 280 papers were submitted for further assessment. Of these, 40 full articles were included in the final evaluation. As a result, 11 papers (full text publications) were incorporated into this review (Figure 1). Although all references from previously identified records were reviewed, no additional sources were identified (other than already found).

We identified 4 studies presenting indirect costs associated with Crohn's disease [8-11], 4 studies for both Crohn's disease and ulcerative colitis [12-15], and 3 studies concerning inflammatory bowel disease in general [16-18], from the following countries: Italy [8], New Zealand [10], Germany [12], United Kingdom [13], Spain [9], Canada [18], Sweden [11, 16], and United States of America [14, 15, 17]. Our systematic review showed that most of the data on Crohn's disease and ulcerative colitis came from Europe.

The major components of indirect costs were: lost productivity due to leaving work earlier because of the disease, absence from work due to sick leave, early retirement or mortality (absenteeism), and loss of leisure time for nonemployees (loss of leisure). A few studies also included costs of travel, carers, additional phone or internet requirements, or special diet, but the above cost categories are not indirect costs. Only one study concerned presenteeism, and none of them concerned unemployment (Tables I and II).
This review included cost assessments from North America and European countries, and New Zealand. In the United States the yearly indirect costs per patient of Crohn's disease and ulcerative colitis including absenteeism were assessed to be $\$ 6434$ and $\$ 7264$, respectively. When only short-term disability was included, the costs were much lower, and amounted to \$1 963 and \$1 673, respectively. Indirect costs assessed in European countries seem to be higher than in the United States. Indirect costs resulting from lost productivity were \$5 128-14 136 for Crohn's disease and \$6 583 for ulcerative colitis. It should be mentioned that more studies with cost data were identified for European countries than for North American countries, and the above conclusion may change when new studies are conducted.

One study carried out in the United States presented total indirect costs for both Crohn's disease and ulcerative colitis. The values ranged from \$1 877 to \$3 754 (work disability). In the review we also included three studies which concerned inflammatory bowel disease in general. One was carried out in Europe (Sweden), one in Canada, and one in the United States. The highest indirect cost (\$7 442) was calculated in the United States, and was due to work loss, whereas the corresponding value for Canada was more than six times smaller. The European study included the indirect cost of inflammatory bowel disease in the

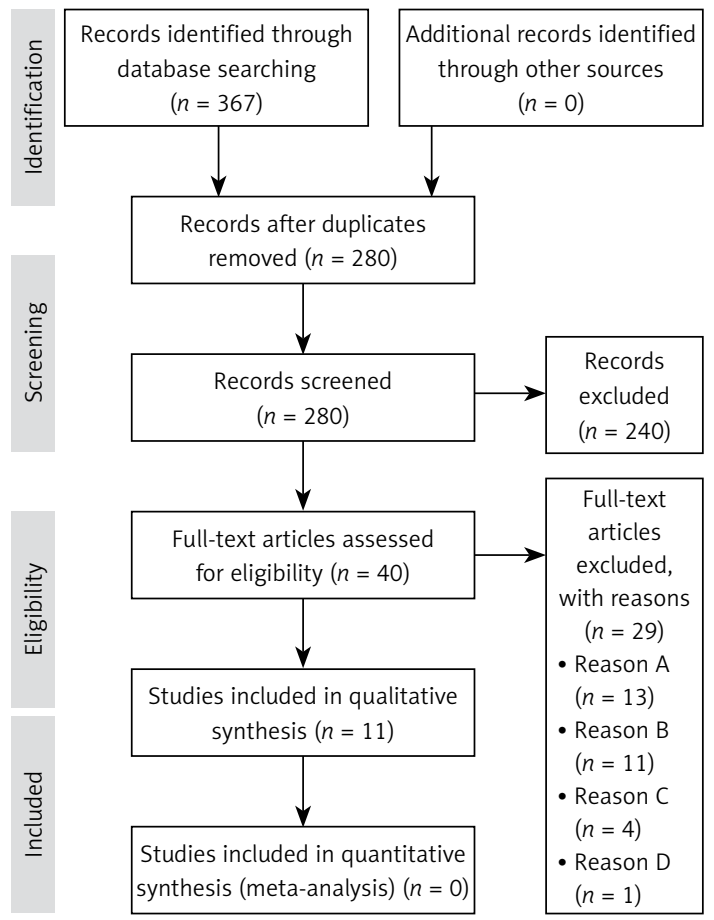

Figure 1. PRISMA flow diagram showing the record selection process. Reason A - secondary data; reason $B$ - different population; reason $C$ - different endpoint; reason $\mathrm{D}$ - other 


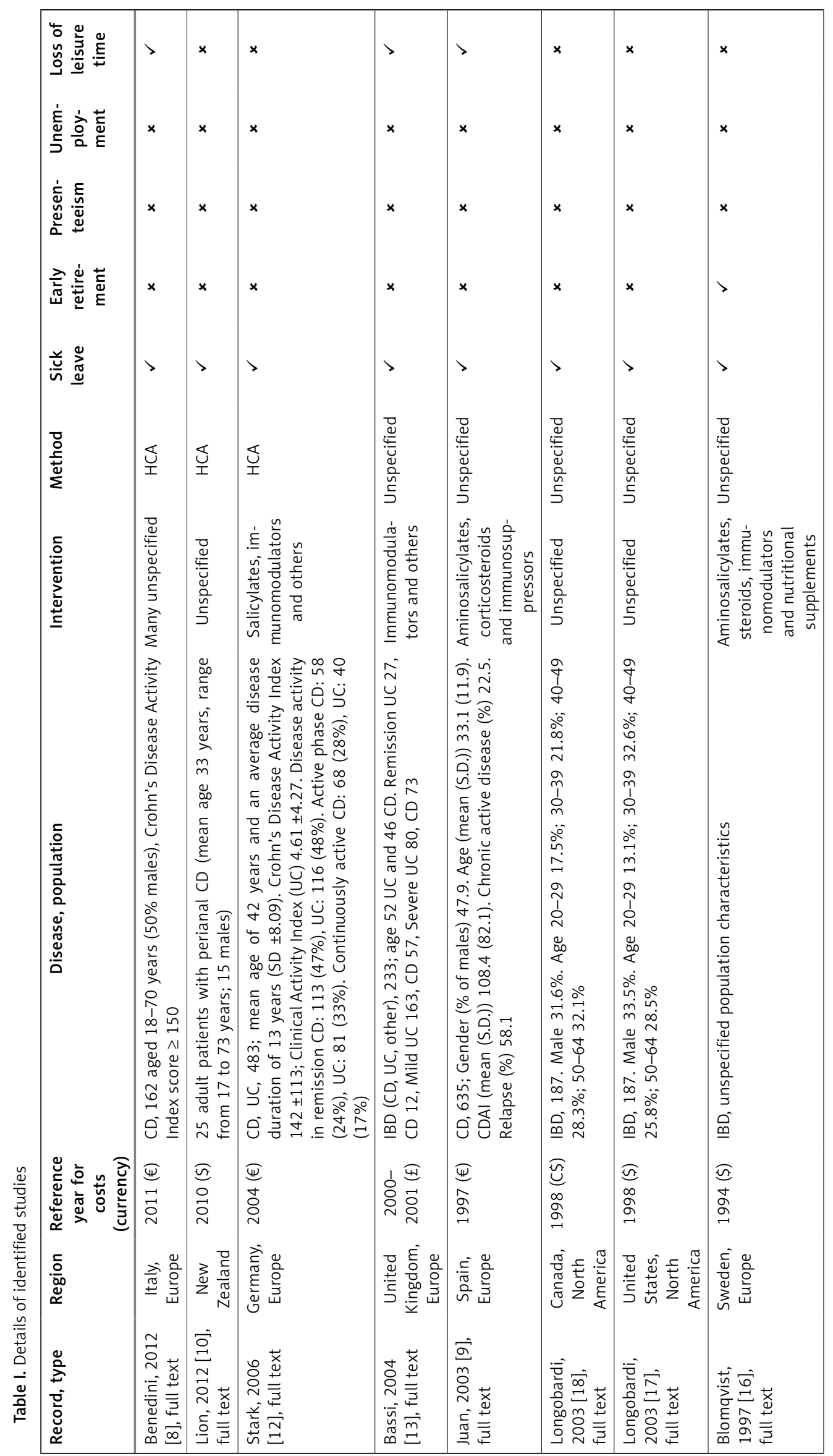


Indirect costs of inflammatory bowel diseases: Crohn's disease and ulcerative colitis. A systematic review

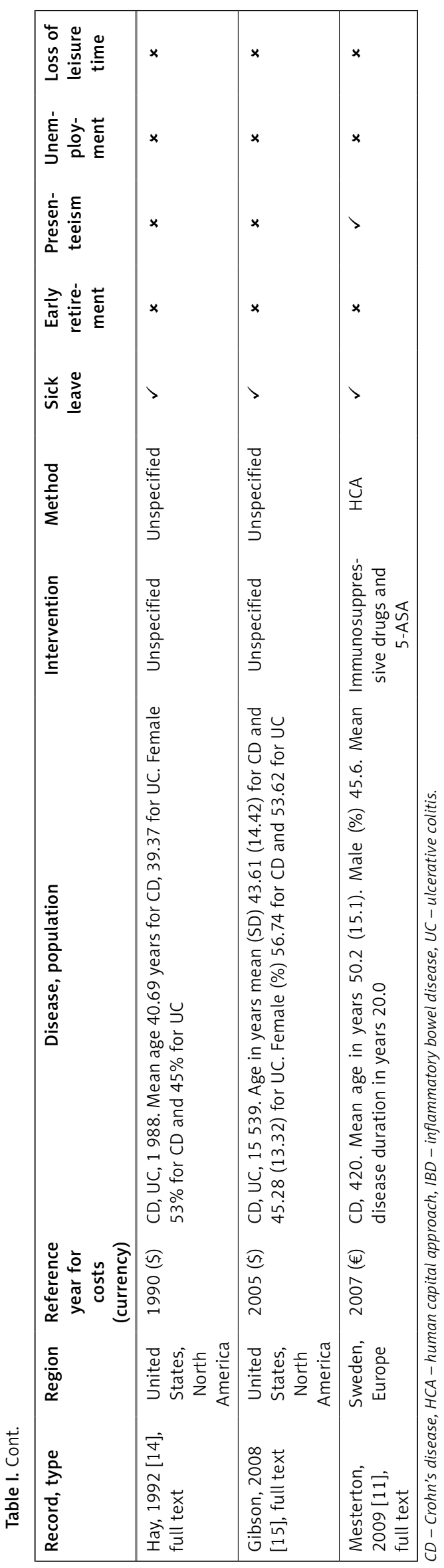




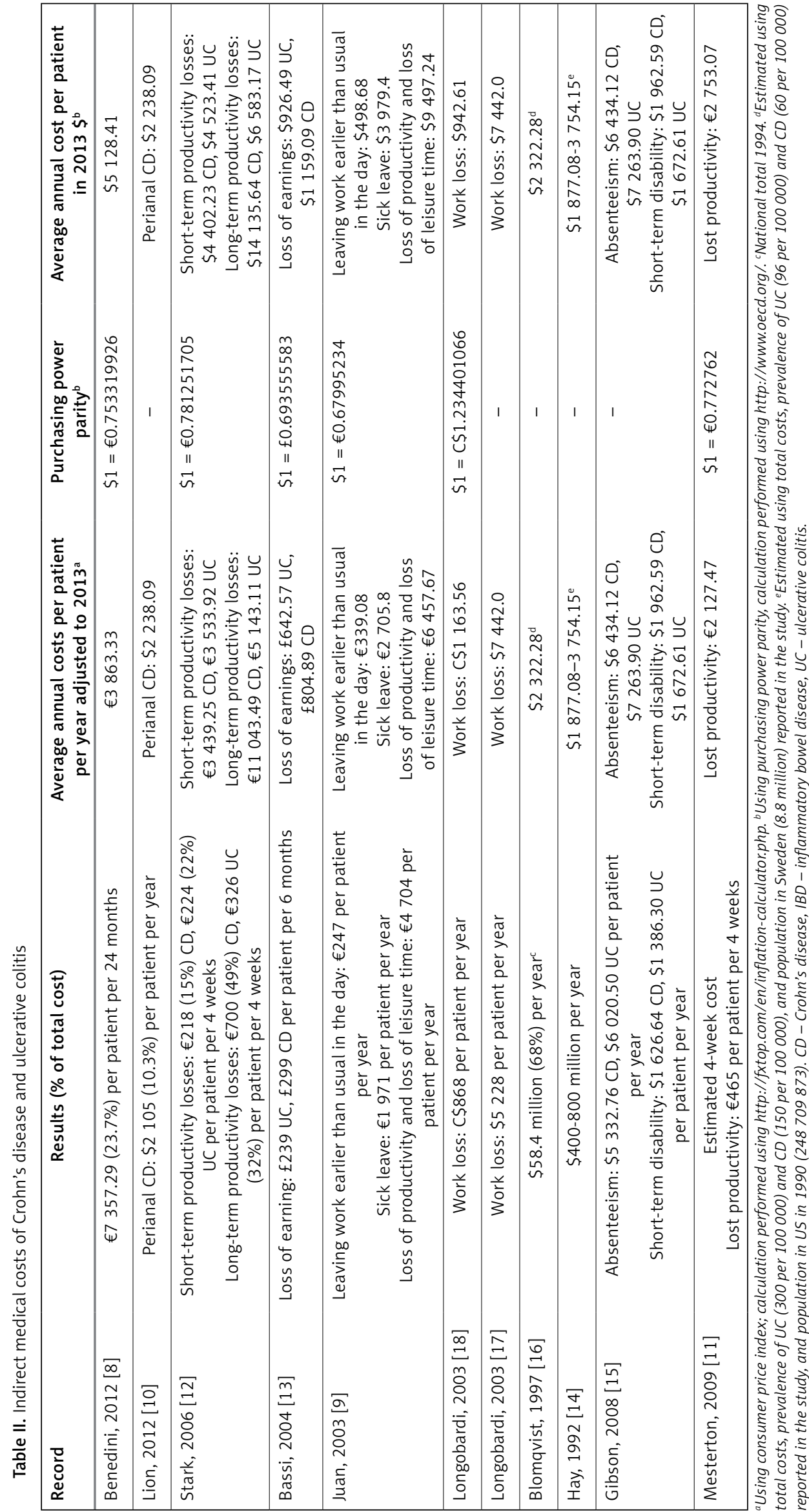


form of sickness leave and early retirement, and it was comparable to the cost assessed in Canada.

The results presented above indicate that the highest component of indirect costs was loss of productivity.

Only the indirect costs that have the same components measured by the same approach, the same population, and endpoints presented as mean and standard deviation can be incorporated into the meta-analysis. Almost all the identified studies reported the numbers of patients used to obtain indirect cost data. In the study by Blomqvist et al. there was only information about national total indirect costs. Only in three studies was the methodology for the estimation of indirect costs specified, and these studies were included in further analysis $[8,10,12]$. The study by Lion et al. [10] was excluded from further research as it includes a slightly different population - paediatric and perianal Crohn's disease patients. The remaining two studies included patients with Crohn's disease, and the study by Stark et al. [12] also reported results for ulcerative colitis patients. The results of the study by Benedini et al. [8] are presented as total indirect costs, without the values for each cost category. Stark et al. [12] presented their results in the form of short- (sick leave) and long-term productivity losses. Taking into account the methodology of the studies $[8,12]$ listed above, and therefore the heterogeneity of the studies identified, there was no possibility to carry out a meta-analysis.

\section{Discussion}

Several studies were identified concerning systematic reviews of the indirect costs of inflammatory bowel disease. In a study by Cohen et al. [19] a systematic review of the costs of ulcerative colitis was conducted. No data were collected for Crohn's disease. The authors included only full texts; abstracts were excluded from the review. All the identified studies were carried out in Europe or North America; no studies from New Zealand were identified. In a study by Yu et al. [20] only the costs of Crohn's disease were collected. The methodology and identified studies were similar to those in the review by Cohen et al. Abstracts and studies beyond North America and Europe were not included in the review. The above reviews were conducted in 2006-2008 and do not include studies from later, e.g. Benedini [8] or Lion [10]. Also, one study published in 2003 by Juan et al. [9] was not included in the identified reviews, but it was described in another review by Odes [21].

Indirect costs identified in this review varied considerably because of the difference in components. Expenses such as loss of earnings due to leaving work earlier, or sick leave, have a minor impact on total indirect expenditures.
The author made an attempt to perform a meta-analysis, but taking into account the differences in the studies' methodology, variety of reported end points and population it was impossible to carry out. In the majority of studies there was no information about the method of indirect cost estimation. Different studies take into account different combinations of indirect cost categories, and there were also differences in the definitions of each category. The above deficiencies and problems need to be resolved in order to improve the decision making process based on the indirect costs of Crohn's disease and ulcerative colitis.

Indirect costs are very difficult to assess, mainly because of a lack of comprehensive data sources and difficulties in measurement. For example, one study included presenteeism, which is the reduction of workers' effectiveness due to illness. Only two studies included loss of leisure time for non-employees as a part of indirect costs. Lion et al. [10] examined the effect of Crohn's disease on costs resulting from travel, carers, tutors, and additional phone and internet requirements, but these cost categories cannot be treated as indirect costs. Also, Bassi et al. [13] assessed the influence of inflammatory bowel disease on out-of-pocket expenses such as travel, prescription charges, special diets, and clothing, which are not indirect costs. It is proved that patients with Crohn's disease have a higher mortality risk than the general population [1]. Nevertheless, none of the identified studies included the cost resulting from increased mortality and hence lost productivity. The above indicates that the identified costs may have been underestimated.

In conclusion, Crohn's disease and ulcerative colitis impose a substantial personal burden and affect the ability to work. The observation concerning the influence of the disease on patients' functional abilities requires special attention in future studies on Crohn's disease and ulcerative colitis. The great variety of methodologies and indirect cost components in studies revealed that it is impossible to carry out a meta-analysis. This review showed that further research is needed due to the lack of information on the topic, and a precise methodology of indirect cost estimation must be developed.

\section{Conflict of interest}

The author declares no conflict of interest.

\section{References}

1. Baumgart DC, Sandborn WJ. Inflammatory bowel disease: clinical aspects and established and evolving therapies. Lancet 2007; 369: 1641-57.

2. Baumgart DC, Sandborn WJ. Crohn's disease. Lancet 2012; 380: 1590-605.

3. Kawalec P, Mikrut A, Wiśniewska N, Pilc A. Tumor necrosis factor-alpha antibodies (infliximab, adalimumab and 
certolizumab) in Crohn's disease: systematic review and meta-analysis. Arch Med Sci 2013; 9: 765-79.

4. Kawalec P, Mikrut A, Łopuch S. Systematic review of the effectiveness of biological therapy for active moderate to severe ulcerative colitis. J Gastroenterol Hepatol 2014; 29: 1159-70.

5. Büsch K, Ludvigsson JF, Ekström-Smedby K, et al. Nationwide prevalence of inflammatory bowel disease in Sweden: a population-based register study. Aliment Pharmacol Ther 2014; 39: 57-68.

6. Nguyen GC, Tuskey A, Dassopoulos T, et al. Rising hospitalization rates for inflammatory bowel disease in the United States between 1998 and 2004. Inflamm Bowel Dis 2007; 13: 1529-35.

7. Krol M, Brouwer W. How to estimate productivity costs in economic evaluations. Pharmacoeconomics 2014; 32: 335-44.

8. Benedini V, Caporaso N, Corazza GR, et al. Burden of Crohn's disease: economics and quality of life aspects in Italy. Clinicoecon Outcomes Res 2012; 4: 209-18.

9. Juan J, Estiarte R, Colome E, et al. Burden of illness of Crohn's disease in Spain. Dig Liver Dis 2003; 35: 853-61.

10. Lion M, Gearry RB, Day AS, et al. The cost of paediatric and perianal Crohn's disease in Canterbury, New Zealand. N Z Med J 2012; 125: 1349.

11. Mesterton J, Jönsson L, Almer SH, Befrits R, Friis-Liby I, Lindgren S. Resource use and societal costs for Crohn's disease in Sweden. Inflamm Bowel Dis 2009; 15: 1882-90.

12. Stark R, Konig HH, Leidl R. Costs of inflammatory bowel disease in Germany. Pharmacoeconomics 2006; 24: 797-814.

13. Bassi A, Dodd S, Williamson P, et al. Cost of illness of inflammatory bowel disease in the UK: a single centre retrospective study. Gut 2004; 53: 1471-8.

14. Hay JW, Hay AR. Inflammatory bowel disease: costsof-illness. J Clin Gastroenterol 1992; 14: 309-17.

15. Gibson TB, Ng E, Ozminkowski RJ, et al. The direct and indirect cost burden of Crohn's disease and ulcerative colitis. J Occup Environm Med 2008; 50: 1261-72.

16. Blomqvist P, Ekbom A. Inflammatory bowel diseases: health care and costs in Sweden in 1994. Scand J Gastroenterol 1997; 32: 1134-9.

17. Longobardi T, Jacobs P, Bernstein CN. Work losses related to IBD in the United States: results from the National Health Interview Survey. Am J Gastroenterol 2003; 98 1064-72.

18. Longobardi T, Jacobs P, Wu L, et al. Work losses related to inflammatory bowel disease in Canada: results from a National Population Health Survey. Am J Gastroenterol 2003; 98: 844-9.

19. Cohen RD, Yu AP, Wu EQ, et al. Systematic review: the costs of ulcerative colitis in Western countries. Aliment Pharmacol Ther 2010; 31: 693-707.

20. Yu AP, Cabanilla LA, Wu EQ, et al. The costs of Crohn's disease in the United States and other Western countries: a systematic review. Curr Med Res Opinion 2008; 24: 319-28.

21. Odes S. How expensive is inflammatory bowel disease? A critical analysis. World J Gastroenterol 2008; 14 6641-7. 